\title{
ABOUT A GENERALIZED ALGEBRAIC RICCATI EQUATION
}

\author{
G. Freiling and A. Hochhaus \\ Department of Mathematics, University of Duisburg, D-47048 Duisburg, Germany \\ freiling@math.uni-duisburg.de, hochhaus@math.uni-duisburg.de
}

\begin{abstract}
We describe the connection between a generalized algebraic Riccati equation and the corresponding generalized algebraic Riccati system and Kalman-Popov-Yakubovich system. Moreover, we present an iterative procedure for constructing its stabilizing solution.
\end{abstract}

Keywords: Generalized algebraic Riccati equation, generalized Lur'e system, generalized Kalman-Popov-Yakubovich system, stabilizing solution.

\section{Introduction}

The solution of stochastic linear quadratic optimal control problems with infinite time horizon leads to algebraic matrix equations of the form (see [3], [18])

$$
\begin{gathered}
A^{*} X+X A+Q+\Pi_{1}(X)- \\
-\left[S+X B+\Pi_{12}(X)\right]\left[R+\Pi_{2}(X)\right]^{+}\left[S+X B+\Pi_{12}(X)\right]^{*}=0,
\end{gathered}
$$

where $Z^{+}$is the Moore-Penrose inverse of a matrix $Z$ and $A, B, Q, R$ and $S$ are given matrices of sizes $n \times n, n \times m, n \times n, m \times m$ and $n \times m$, respectively, such that

$$
T:=\left[\begin{array}{cc}
Q & S \\
S^{*} & R
\end{array}\right]
$$

is hermitian. Moreover, the operator $\Pi: \mathcal{H}^{n} \rightarrow \mathcal{H}^{n+m}$ with

$$
\Pi(X):=\left[\begin{array}{cc}
\Pi_{1}(X) & \Pi_{12}(X) \\
\Pi_{12}(X)^{*} & \Pi_{2}(X)
\end{array}\right]
$$

is linear and positive, i.e. $X \geq 0$ implies $\Pi(X) \geq 0$. Here, $\mathcal{H}^{n}$ stands for the real vector space of hermitian matrices of size $n$, and by $X \geq 0$ (or $X>0$ ) it is denoted that $X=X^{*}$ is positive semidefinite (or positive definite).

The original version of this chapter was revised: The copyright line was incorrect. This has been corrected. The Erratum to this chapter is available at DOI: 10.1007/978-0-387-35690-7_44 
If $R$ is invertible, $S=0$ and $\Pi \equiv 0$ then (1.1) reduces to the wellknown algebraic Riccati equation

$$
A^{*} X+X A+Q-X B R^{-1} B^{*} X=0
$$

which is of great importance in many fields of applied mathematics, e.g. optimal control theory. For that reason we call equation (1.1) generalized algebraic Riccati equation.

For $X \in \mathcal{H}^{n}$ we define the dissipation matrix associated with the generalized algebraic Riccati equation (1.1) as

$$
\Lambda(X):=\left[\begin{array}{cc}
A^{*} X+X A+Q+\Pi_{1}(X) & S+X B+\Pi_{12}(X) \\
{\left[S+X B+\Pi_{12}(X)\right]^{*}} & R+\Pi_{2}(X)
\end{array}\right] .
$$

Below we will describe how equation (1.1) is related to the (continuoustime) generalized algebraic Riccati system (or Luré system)

$$
\Lambda(X)\left[\begin{array}{c}
I \\
K
\end{array}\right]=0
$$

in the unknowns $K \in \mathbb{C}^{m \times n}, X \in \mathcal{H}^{n}$ and to the generalized KalmanPopov-Yakubovich system

$$
\Lambda(X)=\left[\begin{array}{c}
V^{*} \\
W^{*}
\end{array}\right]\left[\begin{array}{ll}
V & W
\end{array}\right]
$$

in the unknowns $X \in \mathcal{H}^{n}, V \in \mathbb{C}^{m \times n}$ and $W \in \mathbb{C}^{m \times m}$.

For the case where $R$ is invertible and $\Pi \equiv 0$ these results can be found in [17]. It turns out that under adequate additional restrictions (see Section 3) (1.1), (1.3) and (1.4) are equivalent.

Finally, in Section 4 we present an iterative procedure for computing the maximal (and stabilizing) solution $X_{+}$of (1.1) which exists under certain generalized stabilizability and detectability assumptions; alternative algorithms for determining $X_{+}$have been developed in [4] (via semidefinite programming associated with LMI's) and in [8], [14] (via Newton-type iteration).

Further results on (1.1) can be found in [2], [4], [5], [6], [7], [8], [9], [10] and [14].

\section{Lyapunov equations and stability}

In this section we present some preliminary results on the linearly perturbed algebraic Lyapunov equation

$$
A^{*} X+X A+\Pi_{1}(X)+Q=0,
$$


where $A$ and $Q$ are given $n \times n$ matrices, $Q$ is hermitian and $\Pi_{1}: \mathcal{H}^{n} \rightarrow$ $\mathcal{H}^{n}$ is a positive linear operator. This equation plays a central role in the analysis of the generalized Riccati equation (1.1).

Define the continuous-time Lyapunov operator $\mathcal{L}_{A}$ by

$$
\mathcal{L}_{A}: \mathcal{H}^{n} \rightarrow \mathcal{H}^{n}, X \mapsto A^{*} X+X A .
$$

For an operator or a matrix $\mathcal{T}$ we denote by $\sigma(\mathcal{T})$ and $r(\mathcal{T})$ its spectrum and its spectral radius, respectively. The open left half-plane is $\mathbb{C}_{-}$.

The first theorem which can be found e.g. in [8] generalizes Lyapunovs stability theorem. A slightly modified version of this result appeared already in [12] (see also [11], Section III); in the case of time-varying coefficients a similar result has been proved in [9], Proposition 4.6.

2.1 Theorem. The following statements are equivalent:

(i) $\sigma(A) \subset \mathbb{C}_{-}$and $r\left(\mathcal{L}_{A}^{-1} \Pi_{1}\right)<1$.

(ii) $-\left(\mathcal{L}_{A}-\Pi_{1}\right)$ is inverse positive, i.e. $-\left(\mathcal{L}_{A}-\Pi_{1}\right)^{-1}$ exists and is a positive operator.

(iii) There is some $X>0$ such that $\left(\mathcal{L}_{A}+\Pi_{1}\right)(X)<0$.

(iv) For any $Q>0$ equation (2.1) has a unique solution $X>0$.

(v) $\sigma\left(\mathcal{L}_{A}+\Pi_{1}\right) \subseteq \mathbb{C}_{-}$.

If any one of these conditions is fulfilled then $A$ is called $c$-stable relative to $\Pi_{1}$.

It turns out that the classical definitions of stabilizability and detectability have to be replaced in our situation by the following generalizations.

2.2 Definition. A pair $(A, B)$ of matrices $A \in \mathbb{C}^{n \times n}$ and $B \in \mathbb{C}^{n \times m}$ is said to be $c$-stabilizable relative to $\Pi$ if there is a matrix $F$ such that $A+B F$ is $c$-stable relative to $\left[\frac{I}{F}\right]^{*} \Pi\left[\begin{array}{l}I \\ F\end{array}\right]$.

According to Theorem $2.1(A, B)$ is $c$-stabilizable relative to $\Pi$ if and only if the inequality $(A+B F)^{*} X+X(A+B F)+\left[\begin{array}{l}I \\ F\end{array}\right]^{*} \Pi(X)\left[\begin{array}{l}I \\ F\end{array}\right]<0$ is fulfilled by a pair $(F, X)$ with $X>0$.

Notice that the concept of mean-square stabilizability used in [12] is in the special case considered therein equivalent to $c$-stabilizability relative to $\Pi$.

2.3 Definition. A pair $(C, A)$ of matrices $A \in \mathbb{C}^{n \times n}$ and $C \in \mathbb{C}^{m \times n}$ is said to be $c$-detectable relative to $\Pi_{1}$ if there is a matrix $L \in \mathbb{C}^{n \times m}$ such that $A+L C$ is $c$-stable relative to $\Pi_{1}$. 
Next we formulate a necessary condition for $c$-detectability which corresponds to the well-known Popov-Belevitch-Hautus criterion.

2.4 Lemma (see [14]). If there exist a positive semidefinite matrix $V \neq 0$ with $C V=0$ and some $\lambda \geq 0$ such that $\left(\mathcal{L}_{A}+\Pi_{1}\right)^{\text {adj }}(V)=\lambda V$, then $(C, A)$ is not c-detectable relative to $\Pi_{1}$.

The following lemma generalizes results known from stability theory in the special case $\Pi_{1} \equiv 0$.

2.5 Lemma (see [14]). Suppose $Q \geq 0$ and (2.1) has a solution $X \geq 0$.

(i) If $Q>0$ then $A$ is c-stable relative to $\Pi_{1}$ and we have $X>0$.

(ii) If $(Q, A)$ is c-detectable relative to $\Pi_{1}$ then $A$ is c-stable relative to $\Pi_{1}$.

\section{Main results}

For convenience of the reader we recall first the definition of the Moore-Penrose inverse and state some of its elementary properties which can be found for example in Section 20.5 of [16].

3.1 Definition. The Moore-Penrose inverse of an $m \times n$ matrix $Z$ is the unique $n \times m$ matrix $Z^{+}$satisfying the conditions

(i) $Z^{+} Z Z^{+}=Z^{+}, Z Z^{+} Z=Z$,

(ii) $\left(Z^{+} Z\right)^{*}=Z^{+} Z,\left(Z Z^{+}\right)^{*}=Z Z^{+}$.

3.2 Lemma. Let $Z$ be an $m \times n$ matrix. Then:

(i) $\left(Z^{+}\right)^{+}=Z$.

(ii) $\left(Z^{*}\right)^{+}=\left(Z^{+}\right)^{*}$.

(iii) $(\lambda Z)^{+}=\lambda^{-1} Z^{+}$for all $\lambda \neq 0$.

(iv) $\operatorname{Ker} Z^{+}=\operatorname{Ker} Z^{*}, \operatorname{Im} Z^{+}=\operatorname{Im} Z^{*}$.

(v) If $Z$ is hermitian or positive semidefinite, then so is $Z^{+}$.

(vi) $Z^{+}=Z^{*}\left(Z Z^{*}\right)^{+}=\left(Z^{*} Z\right)^{+} Z^{*}$.

The following lemma is taken from [1] (see Theorem 9.17 therein):

3.3 Lemma. Assume that $Z$ is an $m \times n$ matrix and $W$ is a $p \times n$ matrix. Then the following statements are equivalent:

(i) $\operatorname{Ker} Z \subseteq \operatorname{Ker} W$.

(ii) $W=W Z^{+} Z$.

(iii) $W^{+}=Z^{+} Z W^{+}$.

For every $X \in \mathcal{H}^{n}$ we introduce the corresponding feedback matrix

$$
F(X):=-\left[R+\Pi_{2}(X)\right]^{+}\left[S+X B+\Pi_{12}(X)\right]^{*} .
$$


The following lemma explains the relation between the generalized algebraic Riccati equation (1.1) and the generalized algebraic Riccati system (1.3).

3.4 Lemma. A matrix $X \in \mathcal{H}^{n}$ is a solution of

$$
\begin{gathered}
A^{*} X+X A+Q+\Pi_{1}(X)-\left[S+X B+\Pi_{12}(X)\right] \\
\times\left[R+\Pi_{2}(X)\right]^{+}\left[S+X B+\Pi_{12}(X)\right]^{*}=0
\end{gathered}
$$

with

$$
\operatorname{Ker}\left[R+\Pi_{2}(X)\right] \subseteq \operatorname{Ker}\left[S+X B+\Pi_{12}(X)\right]
$$

if and only if there is a matrix $K \in \mathbb{C}^{m \times n}$ such that

$$
\Lambda(X)\left[\begin{array}{c}
I \\
K
\end{array}\right]=0 .
$$

In this case $K=F(X)$, if additionally

$$
\operatorname{Ker}\left[R+\Pi_{2}(X)\right] \subseteq \operatorname{Ker} K^{*} .
$$

Proof. (i) If $X \in \mathcal{H}^{n}$ is a solution of (3.1a) and $K:=F(X)$ is the corresponding feedback matrix, it is obvious that

$$
A^{*} X+X A+Q+\Pi_{1}(X)+\left[S+X B+\Pi_{12}(X)\right] K=0 .
$$

Furthermore from Lemma 3.3 it follows that $(3.1 \mathrm{~b})$ is equivalent to

$$
-K^{*}\left[R+\Pi_{2}(X)\right]=S+X B+\Pi_{12}(X),
$$

therefore

$$
\left[S+X B+\Pi_{12}(X)\right]^{*}+\left[R+\Pi_{2}(X)\right] K=0 .
$$

Consequently $K$ satisfies equation (3.2).

(ii) If $X \in \mathcal{H}^{n}$ and $K \in \mathbb{C}^{m \times n}$ are chosen such that (3.2b) holds, then it follows from the properties of the Moore-Penrose inverse that

$$
\begin{aligned}
& {\left[S+X B+\Pi_{12}(X)\right] K=-K^{*}\left[R+\Pi_{2}(X)\right] K} \\
& =-K^{*}\left[R+\Pi_{2}(X)\right]\left[R+\Pi_{2}(X)\right]^{+}\left[R+\Pi_{2}(X)\right] K \\
& =-\left[S+X B+\Pi_{12}(X)\right]\left[R+\Pi_{2}(X)\right]^{+}\left[S+X B+\Pi_{12}(X)\right]^{*} .
\end{aligned}
$$

Plugging this into (3.2a) yields that $X$ satisfies (3.1a). Analogously it is obtained that

$$
\begin{aligned}
& {\left[S+X B+\Pi_{12}(X)\right]\left[R+\Pi_{2}(X)\right]^{+}\left[R+\Pi_{2}(X)\right]} \\
& \quad=-K^{*}\left[R+\Pi_{2}(X)\right]\left[R+\Pi_{2}(X)\right]^{+}\left[R+\Pi_{2}(X)\right] \\
& \quad=-K^{*}\left[R+\Pi_{2}(X)\right]=S+X B+\Pi_{12}(X),
\end{aligned}
$$

and according to Lemma 3.3 this is equivalent to (3.1b). 
(iii) Using parts (i) and (iv) of Lemma 3.2 and Lemma 3.3 it can easily be seen that relation (3.3) is equivalent to $\operatorname{Ker}\left[R+\Pi_{2}(X)\right]^{+} \subseteq$ Ker $K^{*}$ and to $K=\left[R+\Pi_{2}(X)\right]^{+}\left[R+\Pi_{2}(X)\right] K$, respectively. Now premultiplication of $(3.2 \mathrm{~b})$ with $\left[R+\Pi_{2}(X)\right]^{+}$yields the last statement of the lemma.

The next theorem shows the relation between the generalized algebraic Riccati equation (1.1) and the generalized Kalman-Popov-Yakubovich system (1.4); notice that (1.4) provides a factorization of the dissipation matrix (1.2).

3.5 Theorem. A matrix $X \in \mathcal{H}^{n}$ is a solution of

$$
\begin{gathered}
A^{*} X+X A+Q+\Pi_{1}(X)-\left[S+X B+\Pi_{12}(X)\right] \\
\times\left[R+\Pi_{2}(X)\right]^{+}\left[S+X B+\Pi_{12}(X)\right]^{*}=0
\end{gathered}
$$

with

and

$$
\begin{gathered}
\operatorname{Ker}\left[R+\Pi_{2}(X)\right] \subseteq \operatorname{Ker}\left[S+X B+\Pi_{12}(X)\right] \\
R+\Pi_{2}(X) \geq 0,
\end{gathered}
$$

if and only there exist matrices $V \in \mathbb{C}^{m \times n}$ and $W \in \mathbb{C}^{m \times m}$ such that

and

$$
\Lambda(X)=\left[\begin{array}{c}
V^{*} \\
W^{*}
\end{array}\right]\left[\begin{array}{ll}
V & W
\end{array}\right]
$$

In this case $F(X)=-W^{+} V$.

Proof. (i) If $X \in \mathcal{H}^{n}$ is a solution of (3.4) then there exist a matrix $W \in \mathbb{C}^{m \times m}$ with $R+\Pi_{2}(X)=W^{*} W$ and (see Lemma 3.4) a matrix $K \in \mathbb{C}^{m \times n}$ such that (3.2) is fulfilled. Define $V:=-W K$, then

$$
\left[S+X B+\Pi_{12}(X)\right]^{*}=-\left[R+\Pi_{2}(X)\right] K=-W^{*} W K=W^{*} V
$$

and, consequently,

$A^{*} X+X A+Q+\Pi_{1}(X)=-\left[S+X B+\Pi_{12}(X)\right] K=-V^{*} W K=V^{*} V$. Hence $V$ and $W$ satisfy (3.5a). From $V=-W K=-W W^{+} W K=$ $W W^{+} V$ it follows with Lemma 3.3 that $(3.5 \mathrm{~b})$ is valid.

(ii) Let $X \in \mathcal{H}^{n}, V \in \mathbb{C}^{m \times n}$ and $W \in \mathbb{C}^{m \times m}$ be chosen such that (3.5) holds. Then, according to Lemma 3.3, $V=W W^{+} V$. Defining $K:=-W^{+} V$ it follows, using (3.5) again, that

$\left[S+X B+\Pi_{12}(X)\right] K=-V^{*} W W^{+} V=-V^{*} V=-\left[A^{*} X+X A+Q+\Pi_{1}(X)\right]$ and

$$
\left[R+\Pi_{2}(X)\right] K=-W^{*} W W^{+} V=-W^{*} V=-\left[S+X B+\Pi_{12}(X)\right]^{*} .
$$

Therefore $X$ and $K$ satisfy the algebraic Riccati system (3.2), moreover $R+\Pi_{2}(X)=W^{*} W \geq 0$. As a consequence of Lemma $3.4 X$ is a solution of the Kalman-Popov-Yakubovich system (3.5a). Finally, from Lemma 3.2 (vi) we obtain

$$
-W^{+} V=-\left(W^{*} W\right)^{+} W^{*} V=-\left[R+\Pi_{2}(X)\right]^{+}\left[S+X B+\Pi_{12}(X)\right]^{*},
$$

which proves the statement of the theorem. 


\section{Computation of the stabilizing solution}

For the formulation of the subsequent results we define $D(\mathcal{R})$ as the set of all $X \in \mathcal{H}^{n}$ such that

$$
R+\Pi_{2}(X) \geq 0 \quad \text { and } \operatorname{Ker}\left[R+\Pi_{2}(X)\right] \subseteq \operatorname{Ker}\left[S+X B+\Pi_{12}(X)\right]
$$

and the generalized Riccati operator $\mathcal{R}: D(\mathcal{R}) \rightarrow \mathcal{H}^{n}$ by

$$
\begin{gathered}
\mathcal{R}(X)=A^{*} X+X A+Q+\Pi_{1}(X)-\left[S+X B+\Pi_{12}(X)\right] \\
\times\left[R+\Pi_{2}(X)\right]^{+}\left[S+X B+\Pi_{12}(X)\right]^{*} .
\end{gathered}
$$

We agree that all statements concerning solutions $X$ of $\mathcal{R}(X)=0$ are made under the additional hypothesis $X \in D(\mathcal{R})$.

If $X \in \mathcal{H}^{n}$ is a solution of $\mathcal{R}(X)=0$ and $F=F(X)$ denotes the corresponding feedback matrix then $X$ is called stabilizing (resp. almost stabilizing) if $\sigma\left(\mathcal{L}_{A+B F}+\hat{\Pi}\right)$ with $\hat{\Pi}=\left[\begin{array}{l}I \\ F\end{array}\right]^{*} \Pi\left[\begin{array}{l}I \\ F\end{array}\right]$ is contained in the open (resp. closed) left half-plane.

In the following we propose an algorithm for the computation of the stabilizing solution $X_{+}$of $\mathcal{R}(X)=0$ which is based on Theorem 3.5. For convenience below we use the following assumptions which ensure the existence of $X_{+}$:

(H1) $(A, B)$ ist $c$-stabilizable relative to $\Pi$,

(H2) $R>0, T:=\left(\begin{array}{cc}Q & S \\ S^{*} & R\end{array}\right) \geq 0$,

(H3) $\left(Q-S R^{-1} S^{*}, A-B R^{-1} S^{*}\right)$ ist $c$-detectable relative to

$$
\left[\begin{array}{c}
I \\
-R^{-1} S^{*}
\end{array}\right]^{*} \Pi\left[\begin{array}{c}
I \\
-R^{-1} S^{*}
\end{array}\right] \text {. }
$$

The next two lemmata have been proved in [14]:

4.1 Lemma. If $\mathcal{R}(X)=0$ has a stabilizing solution $X_{+}$, then $X_{+} \geq X$ for every solution $X$ of $\mathcal{R}(X) \geq 0$. In particular, $X_{+}$is the (unique) maximal solution of $\mathcal{R}(X)=0$.

4.2 Lemma. Assume that the hypotheses (H2) and (H3) hold. Then every positive semidefinite solution of $\mathcal{R}(X)=0$ is stabilizing.

By hypothesis (H1), there is an $F_{0}$ such that $A_{0}:=A+B F_{0}$ is $c$ stable relative to $\left[\begin{array}{c}I \\ F_{0}\end{array}\right]^{*} \Pi\left[\begin{array}{c}I \\ F_{0}\end{array}\right]$. Therefore, it follows from Theorem 2.1 that the linearly perturbed Lyapunov equation

$$
A_{0}^{*} X_{1}+X_{1} A_{0}+\left[\begin{array}{c}
I \\
F_{0}
\end{array}\right]^{*}\left[T+\Pi\left(X_{1}\right)\right]\left[\begin{array}{c}
I \\
F_{0}
\end{array}\right]=0
$$

has a unique solution $X_{1}$ which is positiv semidefinite since $T \geq 0$. Hence $R+\Pi_{2}\left(X_{1}\right)>0$, and consequently there exist matrices $V_{1} \in$ 
$\mathbb{C}^{m \times n}$ and $W_{1} \in G L(m, \mathbb{C})$ with $R+\Pi_{2}\left(X_{1}\right)=W_{1}^{*} W_{1}$ and $V_{1}^{*} W_{1}=$ $S+X_{1} B+\Pi_{12}\left(X_{1}\right)$. We use induction to construct sequences of matrices $\left\{A_{i}\right\}_{i=0}^{\infty},\left\{F_{i}\right\}_{i=0}^{\infty},\left\{V_{i}\right\}_{i=1}^{\infty},\left\{W_{i}\right\}_{i=1}^{\infty}$ and $\left\{X_{i}\right\}_{i=1}^{\infty}$ with certain properties. Thus, assume that for some $m \geq 1$ we have already determined matrices $\left\{A_{i}\right\}_{i=0}^{m-1},\left\{F_{i}\right\}_{i=0}^{m-1},\left\{V_{i}\right\}_{i=1}^{m},\left\{W_{i}\right\}_{i=1}^{m}$ and $\left\{X_{i}\right\}_{i=1}^{m}$ with

$$
X_{1} \geq X_{2} \geq \ldots \geq X_{m} \geq 0, \quad A_{i}=A+B F_{i}, \quad i=0,1, \ldots, m-1,
$$
where

$$
W_{i}^{*} W_{i}=R+\Pi_{2}\left(X_{i}\right), \quad V_{i}^{*} W_{i}=S+X_{i} B+\Pi_{12}\left(X_{i}\right)
$$

for $i=1,2, \ldots, m$,

$$
\begin{gathered}
F_{i}=-W_{i}^{-1} V_{i}, \quad i=1,2, \ldots, m-1 \\
A_{i}^{*} X_{i+1}+X_{i+1} A_{i}+\left[\begin{array}{c}
I \\
F_{i}
\end{array}\right]^{*}\left[T+\Pi\left(X_{i+1}\right)\right]\left[\begin{array}{c}
I \\
F_{i}
\end{array}\right]=0
\end{gathered}
$$

for $i=0,1, \ldots, m-1$ and the matrices $A_{i}$ are $c$-stable relative to $\left[\begin{array}{c}I \\ F_{i}\end{array}\right]^{*} \Pi\left[\begin{array}{c}I \\ F_{i}\end{array}\right], i=0,1, \ldots, m-1$. Now define $F_{m}:=-W_{m}^{-1} V_{m}$ and $A_{m}:=A+B F_{m}$. Then

$$
\begin{aligned}
& A_{m}^{*} X_{m}+X_{m} A_{m}+\left[\begin{array}{c}
I \\
F_{m}
\end{array}\right]^{*}\left[T+\Pi\left(X_{m}\right)\right]\left[\begin{array}{c}
I \\
F_{m}
\end{array}\right] \\
& \quad+\left(F_{m}-F_{m-1}\right)^{*}\left[R+\Pi_{2}\left(X_{m}\right)\right]\left(F_{m}-F_{m-1}\right)=0
\end{aligned}
$$

and as in the proof of Lemma 4.2 (see [14]) it follows with the hypotheses (H2) and (H3) that $A_{m}$ is $c$-stable relative to $\left[\begin{array}{c}I \\ F_{m}\end{array}\right]^{*} \Pi\left[\begin{array}{c}I \\ F_{m}\end{array}\right]$. According to Theorem 2.1 the linearly perturbed Lyapunov equation

$$
A_{m}^{*} X_{m+1}+X_{m+1} A_{m}+\left[\begin{array}{c}
I \\
F_{m}
\end{array}\right]^{*}\left[T+\Pi\left(X_{m+1}\right)\right]\left[\begin{array}{c}
I \\
F_{m}
\end{array}\right]=0
$$

has a unique solution $X_{m+1} \geq 0$, and the difference $X_{m}-X_{m+1}$ satisfies

$$
\begin{gathered}
A_{m}^{*}\left(X_{m}-X_{m+1}\right)+\left(X_{m}-X_{m+1}\right) A_{m}+\left[\begin{array}{c}
I \\
F_{m}
\end{array}\right]^{*} \Pi\left(X_{m}-X_{m+1}\right)\left[\begin{array}{c}
I \\
F_{m}
\end{array}\right] \\
+\left(F_{m}-F_{m-1}\right)^{*}\left[R+\Pi_{2}\left(X_{m}\right)\right]\left(F_{m}-F_{m-1}\right)=0
\end{gathered}
$$

(see the proof of Theorem 5.2 in [14]). Since $A_{m}$ is $c$-stable relative to $\left[\begin{array}{c}I \\ F_{m}\end{array}\right]^{*} \Pi\left[\begin{array}{c}I \\ F_{m}\end{array}\right]$, it follows from Theorem 2.1 that $X_{m} \geq X_{m+1}$.

So $\left\{X_{i}\right\}_{i=1}^{\infty}$ is a nonincreasing sequence of positiv semidefinite matrices. Hence the limit $X_{+}:=\lim _{i \rightarrow \infty} X_{i}$ exists, and $X_{+} \geq 0$. Furthermore, $X_{+}$satisfies $\mathcal{R}(X)=0$. According to Lemma $4.2 X_{+}$is stabilizing and by Lemma 4.1 also maximal.

A discrete-time version of the algorithm presented above could also be developed (for the basic theory see [13]); in the case $\Pi \equiv 0$ it can be found in [15]. 
4.3 Remark. Note that the maximal solution $X_{+}$also exists (see [14]) if the hypothesis (H1) holds and if (H2) and (H3) are replaced by

(H4) There is a matrix $\hat{X} \in D(\mathcal{R})$ with $\operatorname{Ker}\left[R+\Pi_{2}(\hat{X})\right] \subseteq \operatorname{Ker} B$ for which $\mathcal{R}(\hat{X}) \geq 0$.

Under these weaker assumptions $X_{+}$is at least almost stabilizing and can be computed by replacing (4.1) in the preceding algorithm by

$$
A_{i}^{*} X_{i+1}+X_{i+1} A_{i}+\left[\begin{array}{c}
I \\
F_{i}
\end{array}\right]^{*}\left[T+\Pi\left(X_{i+1}\right)\right]\left[\begin{array}{c}
I \\
F_{i}
\end{array}\right]+\frac{1}{i+1} I=0 ;
$$

or, equivalently, by applying the modified Newton algorithm presented in [14].

Using the theory of the differential equation $-\dot{X}=\mathcal{R}(X)$, developed in [14], we obtain in an elementary way another existence result for $\mathcal{R}(X)=0$.

4.4 Lemma. $\mathcal{R}(X)=0$ has a solution $\hat{X}$ with $\Pi_{2}(\hat{X})>-R$ if and only if there exist $X_{1} \geq X_{2}$ with $\Pi_{2}\left(X_{2}\right)>-R$ and

$$
\mathcal{R}\left(X_{1}\right) \leq 0 \leq \mathcal{R}\left(X_{2}\right) .
$$

Proof. If $\hat{X}$ is a solution of $\mathcal{R}(X)=0$ with $\Pi_{2}(\hat{X})>-R$ then (4.2) holds trivially.

Conversely, it follows from the Monotonicity Lemma 6.1 and the Comparison Theorem 4.5 in [14] that the solutions $X\left(\cdot, X_{1}\right)$ resp. $X\left(\cdot, X_{2}\right)$ of $-\dot{X}=\mathcal{R}(X)$ with $X\left(0, X_{i}\right)=X_{i}, i=1,2$, are decreasing resp. increasing as $t$ is decreasing; moreover

$$
X_{2} \leq X\left(t, X_{2}\right) \leq X\left(t, X_{1}\right) \leq X_{1} \text { for all } t \leq 0 .
$$

Hence the limits

$$
X_{2, \infty}=\lim _{t \rightarrow-\infty} X\left(t, X_{2}\right) \leq X_{1, \infty}=\lim _{t \rightarrow-\infty} X\left(t, X_{1}\right)
$$

exist and solve $\mathcal{R}(X)=0$. Notice that $X\left(t, X_{0}\right)$ exists for $t \leq 0$ if $X_{2} \leq X_{0} \leq X_{1}$.

\section{References}

[1] Ahlbrandt, C. D.; Peterson, A. C.: Discrete Hamiltonian systems, Kluwer Academic Publishers Group, Dordrecht, 1996.

[2] Ait Rami, M.; Chen, X.; Moore, J. B.; Zhou, X. Y.: Solvability and asymptotic behavior of generalized Riccati equations arising in indefinite stochastic LQ controls, IEEE Trans. Automat. Control 46 (2001), 428-440. 
[3] Ait Rami, M.; Moore, J. B.; Zhou, X. Y.: Indefinite stochastic linear quadratic control and generalized differential Riccati equations, Preprint.

[4] Ait Rami, M.; Zhou, X. Y.: Linear matrix inequalities, Riccati equations, and indefinite stochastic linear quadratic controls, IEEE Trans. Automat. Control 45 (2000), 1131-1143.

[5] Chen, S.; Li, X.; Zhou, X. Y.: Stochastic linear quadratic regulators with indefinite control weight costs, SIAM J. Control Optim. 36 (1998), 1685-1702.

[6] Chen, S.; Yong, J.: Stochastic linear quadratic optimal control problems, Appl. Math. Optim. 43 (2001), 21-45.

[7] Chen, S.; Zhou, X. Y.: Stochastic linear quadratic regulators with indefinite control weight costs II, SIAM J. Control Optim. 39 (2000), 1065-1081.

[8] Damm, T.; Hinrichsen, D.: Newton's method for a rational matrix equation occuring in stochastic control, Linear Algebra Appl. 332/334 (2001), 81-109.

[9] Dragan, V.; Morozan, T.: Stability and robust stabilization to linear stochastic systems described by differential equations with markovian jumping and multiplicative white noise, Stochastic Analysis, 20, no.1, 2002, 33-92.

[10] Dragan, V.; Morozan, T.: Systems of matrix rational differential equations arising in connection with linear stochastic systems with markovian jumping, Preprint No. 9/2000, Institutul de Matematică al Academiei Române (2000); to appear in J. Differential Equ., 2003.

[11] Feng, X.; Loparo, K. A.; Ji, Y.; Chizeck, H. J.: Stochastic stability properties of jump linear systems, IEEE Trans. Automat. Control 37 (1992), 38-53.

[12] Fragoso, M. D.; Costa, O. L. V.; de Souza, C. E.: A new approach to linearly perturbed Riccati equations arising in stochastic control, Appl. Math. Optim. 37 (1998), 99-126.

[13] Freiling, G.; Hochhaus, A.: Properties of the solutions of rational matrix difference equations. Advances in difference equations, IV, Comput. Math. Appl., to appear.

[14] Freiling, G.; Hochhaus, A.: On a class of rational matrix differential equations arising in stochastic control, Linear Algebra Appl., to appear.

[15] Halanay, A.; Samuel, J.: Differential equations, discrete systems and control, Kluwer Academic Publishers, Dordrecht, 1997.

[16] Harville, D. A.: Matrix algebra from a statistician's perspective, SpringerVerlag, New York, 1997.

[17] Ionescu, V.; Oară, C.; Weiss, M.: Generalized Riccati theory and robust control, John Wiley \& Sons, Ltd., Chichester, 1999.

[18] Yong, J.; Zhou, X. Y.: Stochastic controls, Springer-Verlag, New York, 1999. 